\title{
SOLUTIONS OF THREE-TERM RELATIONS IN SEVERAL VARIABLES
}

\author{
YUAN XU
}

(Communicated by Palle E. T. Jorgensen)

\begin{abstract}
A system of multivariate orthogonal polynomials satisfies a matrix equation which plays the role of a three-term relation of the orthogonal polynomial of one variable. However, unlike the case of one variable, there does not exist a second solution of this matrix equation that is linearly independent to the orthogonal polynomials. In particular, there is no analogy of the associated polynomials in several variables.
\end{abstract}

\section{INTRODUCTION}

Let $\Pi_{n}^{d}$ be the set of polynomials of total degree $n$ in $d$ variables, and $\Pi^{d}$ be the set of all polynomials in $d$ variables. Let $r_{n}=r_{n}^{d}$ denote $r_{n}=$ $\operatorname{dim} \Pi_{n}^{d}-\operatorname{dim} \Pi_{n-1}^{d}$. For $x_{i} \in \mathbb{R}$ we consider the multiparameter finite difference equations

$$
x_{i} Y_{k}=A_{k, i} Y_{k+1}+B_{k, i} Y_{k}+A_{k-1, i}^{T} Y_{k-1}, \quad 1 \leq i \leq d, k \geq 1,
$$

and the initial values

$$
Y_{0}=a, \quad Y_{1}=\mathbf{b}, \quad a \in \mathbb{R}, \mathbf{b} \in \mathbb{R}^{d},
$$

where $A_{k, i}$ and $B_{k, i}$ are matrices of size $r_{k}^{d} \times r_{k+1}^{d}$ and $r_{k}^{d} \times r_{k}^{d}$, respectively, and $Y_{k} \in \mathbb{R}^{d}$. We assume that the matrices $A_{k, i}$ satisfy a rank condition,

$$
\operatorname{rank} A_{k}=r_{k+1}, \quad A_{k}=\left(A_{k, 1}^{T}|, \ldots,| A_{k, d}^{T}\right)^{T} .
$$

This system of equations appears very naturally in the study of multivariate orthogonal polynomials. Let $\mathscr{L}$ be a linear functional defined on $\Pi^{d}$. Assume $\mathscr{L}(1)=1$ and $\mathscr{L}$ is square positive, i.e., $\mathscr{L}\left(p^{2}\right)>0$ for $p \in \Pi^{d}$. Using the Gram-Schmidt orthogonal process, we have a system of orthonormal polynomials with respect to $\mathscr{L}$, denoted by $\left\{P_{\alpha}^{k}\right\}_{|\alpha|=k=0}^{\infty}$, where $\alpha \in \mathbb{N}_{0}^{d}$ and $P_{\alpha}^{k} \in \Pi_{k}^{d}$. Introducing a vector notation

$$
\mathbb{P}_{k}(\mathbf{x})=\left[P_{\alpha_{1}}^{k}(\mathbf{x}), P_{\alpha_{2}}^{k}(\mathbf{x}), \ldots, P_{\alpha_{r_{k}}}^{k}(\mathbf{x})\right]^{T},
$$

Received by the editors December 3, 1992.

1991 Mathematics Subject Classification. Primary 33C50, 41 A05.

Key words and phrases. Three-term relation, multivariate orthogonal polynomials, associated polynomials. 
where the elements are arranged according to the lexicographical order, we can express the orthonormal property of $\left\{P_{\alpha}^{k}\right\}$ by $\int \mathbb{P}_{k} \mathbb{P}_{m}^{T} W=\delta_{m, k} I$, where $I$ is the identity matrix of size $r_{k} \times r_{k}$. For our convenience, we call $\left\{\mathbb{P}_{k}\right\}_{k=0}^{\infty}$ a sequence of orthonormal polynomials. It is easily seen that $\left\{\mathbb{P}_{k}\right\}$ satisfies equation (1.1) and the initial values

$$
\mathbb{P}_{0}=1, \quad \mathbb{P}_{1}=A_{0}^{-1}\left(\mathbf{x}-B_{0}\right),
$$

where $\mathbf{x}=\left(x_{1}, \ldots, x_{d}\right)^{T}, B_{0}=\left(B_{0,1}, \ldots, B_{0, d}\right)^{T}$. Note that $A_{0}$ is a square matrix; the rank condition (1.3) implies that it is invertible. For $d=1$ equation (1.1) is the classical three-term relation

$$
a_{k} y_{k+1}+b_{k} y_{k}+a_{k-1} y_{k-1}=x y_{k}, \quad k \geq 1,
$$

where $a_{k}>0$. Every system of orthonormal polynomials in one variable satisfies a difference equation (1.4) and the initial conditions

$$
y_{0}=1, \quad y_{1}=a_{0}^{-1}\left(x-b_{0}\right) .
$$

It is well known that (1.4) has another solution which corresponds to the initial conditions

$$
y_{0}=0, \quad y_{1}=a_{0}^{-1} \text {. }
$$

This solution, denoted by $\left\{q_{k}\right\}$, is customarily called the solution of associated polynomials, or polynomials of the second kind. Together, these two solutions of (1.4) share many interesting properties, and $q_{n}$ plays a very important role in areas such as problems of moment, the spectral theory of the Jacobi matrix, Páde approximations, and continuous fractions (cf. $[1,2,5])$.

Inspired by the success and importance of the associated polynomials, we naturally look for their generalization in several variables, and expect that other linearly independent solutions of (1.1) should exist and play the role. However, it turns out surprisingly that (1.2) has no other solutions apart from the system of orthogonal polynomials. This is the main result of this paper, which we formulate as follows.

Theorem 1. If the multiparameter difference equation (1.1) has solution $\mathbb{P}=$ $\left\{\mathbb{P}_{k}\right\}_{k=0}^{\infty}$ for the particular initial value

$$
Y_{0}^{*}=1, \quad Y_{1}^{*}=A_{0}^{-1}\left(\mathbf{x}-B_{0}\right),
$$

then all other solutions of (1.1) and (1.2) are multiples of $\mathbb{P}$ with the possible exception of the first component. More precisely, if $Y=\left\{Y_{k}\right\}_{k=0}^{\infty}$ is a solution of (1.1) and (1.2), then $Y_{k}=h \mathbb{P}_{k}$ for all $k \geq 1$, where $h$ is a function independent of $k$.

This result reflects an essential difference between orthogonal polynomials of one variable and several variables. It shows that there is no analogy of the associated polynomials in several variables. There is another way of formulating this problem. The solution of $(1.1)$ can be viewed as joint eigenvectors of a family of linear operators defined on $l^{2}$. These operators are defined as block Jacobi matrices, i.e., block tridiagonal matrices, with $B_{k, i}$ on the main 
diagonal and $A_{k, i}$ on the subdiagonals. The structure of (1.1) allows us to use the operator theory for a commuting family of selfadjoint operators to study the joint spectrum of the block Jacobi matrices (see $[7,8,9])$. From this point of view, Theorem 1 reflects the fact that the joint spectrum is much more stringent.

\section{ProOF}

For properties of multivariate orthogonal polynomials we refer to $[3,4,6-9]$ and the references therein. Here we need Favard's theorem.

Theorem 2. Let $\left\{\mathbb{P}_{k}\right\}_{k=0}^{\infty}, \mathbb{P}_{0}=1$, be a sequence in $\Pi^{d}$. Then the following statements are equivalent:

(1) There exists a linear functional which is square positive on $\Pi^{d}$ and makes $\left\{\mathbb{P}_{k}\right\}_{k=0}^{\infty}$ an orthonormal basis in $\Pi^{d}$.

(2) For $k \geq 0,1 \leq i \leq d$, there exist matrices $A_{k, i}: r_{k} \times r_{k+1}$ and $B_{k, i}: r_{k} \times r_{k}$, such that the $\mathbb{P}_{k}$ 's satisfy (1.1), (1.5), and the rank condition (1.3).

This theorem is proved in its present form in [6, 7]; it improves the earlier version of Kowalski [4] by using the rank condition. From this theorem, we have that the solution $\mathbb{P}$ in Theorem 1 is orthogonal with respect to a square linear functional $\mathscr{L}$. Since the three-term relation allows us to compute the matrices $\mathscr{L}\left(x_{i} \mathbb{P}_{k} \mathbb{P}_{m}^{T}\right), m=k-1, k, k+1$, in two different ways, we have that the coefficient matrices of (1.1) satisfy

$$
A_{k, i} B_{k+1, j}+B_{k, i} A_{k, j}=B_{k, j} A_{k, i}+A_{k, j} B_{k+1, i},
$$

$$
A_{k-1, i}^{T} A_{k-1, j}+B_{k, i} B_{k, j}+A_{k, i} A_{k, j}^{T}=A_{k-1, j}^{T} A_{k-1, i}+B_{k, j} B_{k, i}+A_{k, j} A_{k, i}^{T},
$$

for $1 \leq i, j \leq d$. We call these equations commuting ones, because they are the conditions that make the block Jacobi matrices formally commuting. From the rank condition (1.3) the generalized inverse of $A_{k}$ exists, we denote it by $D_{k}^{T}=\left(D_{k, 1}|\cdots| D_{k, d}\right)$. Then

$$
D_{k}^{T} A_{k}=\sum_{i=1}^{d} D_{k, i}^{T} A_{k, i}=I .
$$

Using the generalized inverse, we see that every solution of (1.1) satisfies

$$
Y_{k+1}=\sum_{i=1}^{d} D_{k, i}^{T} x_{i} Y_{k}-E_{k} Y_{k}-F_{k} Y_{k-1},
$$

where

$$
E_{k}=\sum_{i=1}^{d} D_{k, i}^{T} B_{k, i}, \quad F_{k}=\sum_{i=1}^{d} D_{k, i}^{T} A_{k-1, i}^{T}
$$


We now prove Theorem 1. The assumption and Favard's theorem imply that $\mathbb{P}=\left\{\mathbb{P}_{k}\right\}_{k=0}^{\infty}$ forms a sequence of orthonormal polynomials. Therefore, the coefficient matrices of (1.1) satisfy (2.1)-(2.3).

Suppose a sequence of vectors $\left\{Y_{k}\right\}$ satisfies (1.1) and initial values (1.2). From (1.1) we have

$$
A_{k, i} Y_{k+1}=x_{i} Y_{k}-B_{k, i} Y_{k}-A_{k-1, i}^{T} Y_{k-1}, \quad 1 \leq i \leq d .
$$

Multiplying the $i$ th equation by $A_{k-1, j}$ and $j$ th equation by $A_{k-1, i}$, we obtain from (2.1) that

$$
A_{k-1, i}\left(x_{j} Y_{k}-B_{k, j} Y_{k}-A_{k-1, j}^{T} Y_{k-1}\right)=A_{k-1, j}\left(x_{i} Y_{k}-B_{k, i} Y_{k}-A_{k-1, i}^{T} Y_{k-1}\right) \text {, }
$$

for $1 \leq i, j \leq d$, and $k \geq 1$. In particular, the case $k=1$ means that $Y_{0}$ and $Y_{1}$ have to satisfy

$$
A_{0, i}\left(x_{j} Y_{1}-B_{0, j} Y_{1}-A_{0, j}^{T} Y_{0}\right)=A_{0, j}\left(x_{i} Y_{1}-B_{0, i} Y_{1}-A_{0, i}^{T} Y_{0}\right)
$$

for $1 \leq i, j \leq d$. Since $A_{0, i} A_{0, j}^{T}$ and $B_{0, i}$ are numbers, and by (2.2) for $k=1$

$$
A_{0, i} B_{1, j}-A_{0, j} B_{1, i}=A_{0, i} B_{0, j}-A_{0, j} B_{0, i},
$$

we can rewrite $(2.8)$ as

$$
\left(x_{i}-B_{0, i}\right) A_{0, j} Y_{1}=\left(x_{j}-B_{0, j}\right) A_{0, i} Y_{1} .
$$

However, $x_{i}$ and $x_{j}$ are independent variables. We see that $Y_{1}$ must be a function of $\mathbf{x}$; moreover, it has to satisfy

$$
A_{0, i} Y_{1}=\left(x_{i}-b_{i}\right) h(\mathbf{x}),
$$

where $h$ is a function of $\mathbf{x}$. Substituting (2.9) into (2.8), we obtain that $b_{i}=$ $B_{0, i}$. The case $h(\mathbf{x})=1$ corresponds to the orthogonal polynomial solution $\mathbb{P}$. Since $D_{0}^{T}=A_{0}^{-1}$, we have from (1.5)

$$
Y_{1}=\sum_{i=1}^{d} D_{0, i}^{T}\left(x_{i}+B_{0, i}\right) h(\mathbf{x})=A_{0}^{-1}\left(\mathbf{x}+B_{0}\right) h(\mathbf{x})=h(\mathbf{x}) \mathbb{P}_{1} .
$$

Therefore, from (2.5),

$$
\begin{aligned}
Y_{2} & =\sum_{i=1}^{d} D_{1, i}^{T}\left(x_{i} I-B_{1, i}\right) h(\mathbf{x}) \mathbb{P}_{1}-F_{1} Y_{0} \\
& =h(\mathbf{x}) \mathbb{P}_{2}+F_{1}\left(h(\mathbf{x})-Y_{0}\right) .
\end{aligned}
$$

Since $\mathbb{P}$ is a solution of the equation (1.1) with the initial condition (1.5), we have from (2.7) that

$$
A_{1, i}\left(x_{j} \mathbb{P}_{2}-B_{2, j} \mathbb{P}_{2}-A_{1, j}^{T} \mathbb{P}_{1}\right)=A_{1, j}\left(x_{i} \mathbb{P}_{2}-B_{2, i} \mathbb{P}_{2}-A_{1, i}^{T} \mathbb{P}_{1}\right) \text {. }
$$

Using this equation and the formulas we derived for $Y_{1}$ and $Y_{2}$, we obtain from (2.7) with $k=2$ that

$$
A_{1, i}\left(x_{j} I-B_{2, j}\right) F_{1}\left(h(\mathbf{x})-Y_{0}\right)=A_{1, j}\left(x_{i} I-B_{2, i}\right) F_{1}\left(h(\mathbf{x})-Y_{0}\right) .
$$


If $Y_{0}=h(\mathbf{x})$, then we have from (2.5) and (2.10) that $Y_{k}=h(\mathbf{x}) \mathbb{P}_{k}$ for all $k \geq 0$, which is the conclusion of the theorem. We now assume that $h(\mathbf{x}) \neq Y_{0}$. Thus, $h(\mathbf{x})-Y_{0}$ is a nonzero number, and we have from the previous formula

$$
A_{1, i}\left(x_{j} I-B_{2, j}\right) F_{1}=A_{1, j}\left(x_{j} I-B_{2, i}\right) F_{1} \text {. }
$$

However, $x_{i}$ and $x_{j}$ are independent variables. We conclude that for this equality to hold it is necessary that $A_{1, i} F_{1}=0$, which implies $F_{1}=0$ by (2.4). We then obtain from (2.11) that $Y_{2}=h(\mathbf{x}) \mathbb{P}_{2}$. Thus, by (2.5) and (2.10) we have $Y_{k}=h(\mathbf{x}) \mathbb{P}_{k}$ for all $k \geq 1$, which concludes the proof of Theorem 1 .

We note that if $F_{1}$ is not zero, then we must have $Y_{0}=h(\mathbf{x})$ as well. Although $F_{1} \neq 0$ can be verified in many cases, for example, bivariate orthogonal polynomials on the product region, it seems to be likely that $F_{1}=0$ may hold for some particular cases.

\section{REFERENCES}

1. N. I. Akheizer, The classical moment problem and some related questions in analysis, Hafner, New York, 1965.

2. T. S. Chihara, An introduction to orthogonal polynomials, Mathematics and its Applications, vol. 13, Gordon \& Breach, New York, 1978.

3. M. A. Kowalski, The recursion formulas for orthogonal polynomials in $n$ variables, SIAM J. Math. Anal. 13 (1982), 309-315.

4. __ Orthogonality and recursion formulas for polynomials in $n$ variables, SIAM J. Math. Anal. 13 (1982), 316-323.

5. M. Stone, Linear transformations in Hilbert space and their applications to analysis, Amer. Math. Soc. Colloq. Publ., vol. 15, Amer. Math. Soc., Providence, RI, 1932; reprinted, 1983.

6. Y. Xu, On multivariate orthogonal polynomials, SIAM J. Math. Anal. 24 (1993), 783-794.

7. _ Multivariate orthogonal polynomials and operator theory, Trans. Amer. Math. Soc. (to appear).

8. _ Unbounded commuting operators and multivariate orthogonal polynomials, Proc. Amer. Math. Soc. 119 (1993), 1223-1231.

9. Math. Soc. (to appear).

Department of Mathematics, University of Oregon, Eugene, Oregon 97403-1222

E-mail address: yuan@bright.uoregon.edu 